\title{
MINIMAL ENTROPY CONDITIONS FOR BURGERS EQUATION
}

\author{
BY \\ CAMILLO DE LELLIS (Max-Planck Institute for Mathematics in the Sciences, Inselstr. 22, \\ D-04103 Leipzig, Germany). \\ FELIX OTTO (Institute for Applied Mathematics, University of Bonn, Wegelerstr. 10, D-53115 \\ Bonn, Germany). \\ A.ND
}

MICHAEL WESTDICKENBERG (Département de mathématiques et applications, Ecole Normale Supérieure, 45 rue d'Ulm, F-75230 Paris cedex 05, France)

Abstract. We consider uniformly convex, 1-d scalar conservation laws. We show that a single uniformly convex entropy is sufficient to characterize a Kruzhkov solution. The proof uses the concept of viscosity solution for the related Hamilton-Jacobi equation.

1. Introduction. We consider a scalar conservation law in one space dimension with uniformly convex flux $f \in C^{2}(\mathbf{R})$

$$
u_{t}+f(u)_{x}=0 \quad \text { for }(t, x) \in[0, \infty) \times \mathbf{R}
$$

and initial data $u(0, \cdot)=u_{0} \in L^{\infty}(\mathbf{R})$. It is well known that, even for smooth initial data, the solution of (1.1) ceases to exist in the classical sense due to the formation of shocks in finite time (cf. Riemann 1859 [10]). Therefore one has to consider weak solutions, i.e., functions with suitable integrability, satisfying (1.1) in distributional sense. Since weak solutions lack uniqueness, additional assumptions must be imposed to select the (physically) relevant ones.

In 1957, Oleinik [8] proved existence and uniqueness of bounded weak solutions of (1.1) which satisfy her "condition $E$ ": She proved that the one-sided Lipschitz condition

$$
\left\|\left(u_{x}\right)_{+}(t, \cdot)\right\|_{L^{\infty}(\mathbf{R})} \leq \frac{1}{c t} \text { for all } t>0
$$

\footnotetext{
Received July, 2003.

2000 Mathematics Subject Classification. Primary 35L65; Secondary 35L67.

Key words and phrases. Entropy solutions, entropy conditions, viscosity solutions.

The authors acknowledge partial support by the EU Network Hyperbolic and kinetic equations HPRNCT-2002-00282.

E-mail address: delellis@mis.mpg.de

E-mail address: otto@iam.uni-bonn.de

E-mail address: westdick@dma.ens.fr
} 
where $c:=\inf f^{\prime \prime}$, singles out one particular among all possible weak solutions. Note that (1.2) only allows for decreasing jumps. As an immediate consequence of (1.2) we obtain a smoothing effect: Initial data in $L^{x}$ are instantancously regularized to $B V_{l o c}$. Additional nice properties follow from (1.2), such as regularity of solutions, rates of convergence to limiting profiles as $t \rightarrow \infty$, convergence of approximation schemes, etc. We refer to Sec. 11 of [4].

A more flexible approach to scalar conservation laws. feasible also in higher space dimensions and for nonconvex fluxes, was given by Kruzhkov [6] in 1970, following earlier work by Conway and Smoller and Vol'pert. For initial data in $L^{\infty}$. Kruzhkov proved existence and uniqueness of bounded weak solutions satisfying the following entropy condition: He considers the family of convex entropy -entropy flux pairs $\left\{\left(\eta_{k}, q_{k}\right)\right\}_{k \in \mathbf{R}}$. where

$$
\eta_{k \cdot}(u):=|u-k| \text { and } q_{k}(u):=\operatorname{sgn}(u-k)(f(u)-f(k)) .
$$

Then an entropy solution is a bounded function $u$ satisfying (1.1) in distributional scuse and

$$
\eta(u)_{t}+q(u)_{x} \leq 0 \text { in } \mathcal{D}^{\prime}
$$

for all $(\eta . q) \in\left\{\left(\eta_{k} . q_{k}\right)\right\}_{k \in \mathbf{R}}$. Equivalently, we may assume (1.3) for all convex functions (entropies) $\eta$. with corresponding entropy flux $q$ defined by $q^{\prime}=\eta^{\prime} f^{\prime}$. Kruzhkov s proof is based on the observation that the solution operator of $(1.1)$ is. in fact, an $L^{1}$-contraction. As a consequence of this. if initial data are in $B V_{l o c}$. then $u \in B V_{l o c}$ for all later times.

One can show that for convex flux. the two entropy conditions (1.2) and (1.3) are equivalent. Hence Oleinik's "condition E" - and Kruzhkov's entropy solutions coincide. It has been an important open question whether a restricted entropy condition. i.e.. assuming (1.3) only for a subset of convex cutropy cutropy flux pairs. would enforce uniqueness of the solution (and hence provide us with all the nice features of Oleinik's solutions). This question is also quite interesting in view of the fact that for most systems of conservation laws. only few convex entropies or even just one (the physical) entropy - are known to exist.

The problem has been solved first in a paper by Panov [9], who proves that if $f$ is uniformly convex. then assimption (1.3) for one single uniformly convex entropy-entropy flux pair $(\eta, q)$ is sufficient to establish Oleinik's "condition $E^{*}$. and thereby characterize entropy solutions among all bounded weak solutions of (1.1). Thus it is not necessary to consider the whole family of convex entropies, or all Kruzhkov entropies. One single pair $(\eta, q)$, with $\eta$ uniformly convex, gives all the information.

In this paper, we give a new proof of this result: see Corollary 2.5. Like Panov, we use the theory of Hamilton-Jacobi equations. But our argument is different from his, and more general for three reasons: First. our approach is not restricted to the Cauchy problem on the real line. Second, we can allow a right-hand side in the entropy inequality: Third, our proof also works for unbounded functions.

The last generalization is important since it allows for the derivation of new estimates for the Kuramoto-Shivashinsky equation; see [5]. We prove that for Burgers flux $f(u)=$ $\frac{1}{2} u^{2}$, and for the special convex entropy $\eta(u):=\frac{1}{2} u^{2}$ (with corresponding entropy flux $\left.q(u):=\frac{1}{3} u^{3}\right)$, it is sufficient to require $u \in L_{\text {loc }}^{4}$ only, instead of $u \in L^{\infty}$. Then still 
a restricted entropy information is sufficient to single out entropy solutions of Burgers equation; see Corollary 2.5 below. We believe that this result can be generalized, allowing for different strictly convex fluxes and entropies. Then the optimal integrability of $u$ should depend on the growth rates of $f$ and $\eta$ at infinity.

As mentioned above, our proof is based on the relation between scalar conservation laws in one space dimension and Hamilton Jacobi equations. Integrating (1.1) in $x$, we obtain

$$
h_{t}+f\left(h_{x}\right)=0 \text { for }(t, x) \in[0, \infty) \times \mathbf{R},
$$

where $h$ is the $x$ primitive of $u$. i.e., $h_{x}=u$. Therefore, (1.1) and (1.4) are formally equivalent. There exists a rather complete existence and uniqueness theory for (1.4) based on the concept of viscosity solutions, first introduced by Crandall and Lions in [3] (see also [7]). It is well known that, when $f$ is strictly convex, $h$ is a viscosity solution of (1.4) if and only if $u=h_{x}$ is an cntropy solution of (1.1). In Theorems 2.3 and 2.4 we prove that the assumptions on $u$ are sufficient to guarantee that $h$ is a viscosity solution of (1.4).

Our proof has been inspired by a recent result of Ambrosio, Lecumberry, and Rivière [2]. There the authors proved that, if $h \in W_{l o c}^{1 . x}\left(\mathbf{R}^{2}\right)$ solves the eikonal equation, then a certain one parameter family of entropy conditions on $\nabla h$ is sufficient to ensure that $h$ is a viscosity solution. As in that paper, our proofs rely on commutator estimates, which are similar in spirit to the arguments used in compensated compactness theory.

2. Main result. We begin by giving the definitions of entropy solutions of scalar conservation laws and viscosity solutions of Hamilton-Jacobi equations.

Definition 2.1. Let $\Omega \subset \mathbf{R}^{2}$ and $f \in W_{l o c}^{1 . \infty}(\mathbf{R})$. We say that $\eta, q \in W_{l o c}^{1, \times}(\mathbf{R})$ is a convex entropy-entropy flux pair if $\eta$ is convex and $q^{\prime}=f^{\prime} \eta^{\prime}$ almost everywhere. A function $u \in L_{l o c}^{1}(\Omega)$ is called an entropy solution of $u_{t}+f(u)_{x}=0$ if

$$
\begin{aligned}
u_{t}+f(u)_{x} & =0 \quad \text { in } \mathcal{D}^{\prime}(\Omega) . \\
\eta(u)_{t}+q(u)_{x} & \leq 0 \quad \text { in } \mathcal{D}^{\prime}(\Omega)
\end{aligned}
$$

for every convex entropy cutropy flux pair $(\eta, q)$.

Definition 2.2. A function $h \in C(\Omega)$ is called a viscosity solution of $h_{t}+f\left(h_{x}\right)=0$ if, for any $(t, x) \in \Omega$, the following hold:

(1) If $\zeta$ is any smooth function such that $h-\zeta$ has a local maximum at $(t, x)$, then

$$
\zeta_{t}(t, x)+f\left(\zeta_{x}(t, x)\right) \leq 0 .
$$

(2) If $\zeta$ is any smooth function such that $h-\zeta$ has a local minimum at $(t, x)$, then

$$
\zeta_{t}(t, x)+f\left(\zeta_{x}(t, x)\right) \geq 0 .
$$

A function $h$ for which (1) (resp. (2)) holds is called a viscosity sub/supersolution.

We are now ready to state our results: 
THEOREM 2.3. Let $f \in C^{2}(\mathbf{R})$ be uniformly convex and fix an entropy-entropy flux pair $(\eta, q)$ with $\eta$ uniformly convex. Assume that $\Omega \subset \mathbf{R}^{2}$ is an open set and $u \in L^{\infty}(\Omega)$ satisfies

$$
\begin{aligned}
u_{t}+f(u)_{x} & =0 \quad \text { in } \mathcal{D}^{\prime}(\Omega), \\
\eta(u)_{t}+q(u)_{x} & \leq 0 \text { in } \mathcal{D}^{\prime}(\Omega) .
\end{aligned}
$$

If $h$ is a function with $h_{t}=-f(u)$ and $h_{x}=u$. then $h$ is a viscosity solution of $h_{t}+f\left(h_{x}\right)=$ 0 .

For Burgers equation we even have

Theoren 2.4. Let $\Omega \subset \mathbf{R}^{2}$ be open, and assume that the function $u \in L_{\text {loc }}^{4}(\Omega)$ satisfies

$$
\begin{aligned}
u_{t}+\left(\frac{u^{2}}{2}\right)_{. r} & =0 \quad \text { in } \mathcal{D}^{\prime}(\Omega) . \\
\left(\frac{u^{2}}{2}\right)_{t}+\left(\frac{u^{3}}{3}\right)_{x} & \leq \mu \quad \text { in } \mathcal{D}^{\prime}(\Omega)
\end{aligned}
$$

for some non-negative Radon measure $\mu$ with

$$
\lim _{r \mid 0} \frac{\mu\left(B_{r}(t, x)\right)}{r}=0 \quad \text { for every }(t, x) \in \Omega \text {. }
$$

If $h$ is a function with $h_{t}=-\frac{u^{2}}{2}$ and $h_{x}=u$. then $h$ is a viscosity solution of $h_{t}+\left(\frac{h_{r}^{2}}{2}\right)=0$.

We observe that condition (2.7) on the measure $\mu$ is optimal. It is just enough to rule out the presence of undercompressive shocks in $(t, r)$, in which case

$$
\liminf _{r l 0} \frac{\mu\left(B_{r}(t . x)\right)}{r}>0 .
$$

The first theorem is proved in Sec. 4 and the second is proved in Sec. 5. They both rely on some elementary inequalities on averages of functions, which are proved in Sec. 3. As a consequence of the theorems above we obtain:

COROLlary 2.5. The function $u$ of Theorem 2.3 is an entropy solution of (2.3). Similarly, the function $u$ of Theorem 2.4 is an entropy solution of $(2.5)$.

Proof. As already mentioned in the introduction, it is a well-known fact that $h$ is a viscosity solution of $h_{t}+f\left(h_{x}\right)=0$ if and only if $u=h_{x}$ is an entropy solution of $u_{t}+f(u)_{t}=0$. To prove this we can use, for instance, the Hopf-Lax formula, which gives explicitly the viscosity solution to $h_{t}+f\left(h_{x}\right)=0$ in terms of $h(0, \cdot)$. We here give an alternative proof, which uses the properties of viscosity solutions to show that $u=h_{x} \in B V_{l o c}$; for $B V$ functions it can be shown directly that $u$ meets the shockadmissibility criterion which distinguishes entropy solutions.

First Step. $B V$ regularity.

In the case of Theorem 2.3, since $h$ is locally Lipschitz and is a viscosity solution of $h_{t}+f\left(h_{x}\right)$, Corollary 9.2 of [7] gives that for every open $U \subset \subset \Omega$, there exists a constant $C$ such that $h_{x x} \leq C$ in the sense of distributions. Differentiating $h_{t}+f\left(h_{x}\right)=0$ with 
respect to $x$, we get that $h_{x t}=h_{t x}=-\left(f\left(h_{x}\right)\right)_{x}$ is also a measure. Hence $u=h_{x} \in$ $B V_{\text {loc }}\left(\mathbf{R}^{2}\right)$.

In the case of Theorem 2.4, a standard comparison argument with supersolutions of type $k \psi(t)\left|x-x_{0}\right|$ gives that $h_{x}$ is locally bounded. Thus $h_{t}=-\frac{\left(h_{x}\right)^{2}}{2}$ is locally bounded as well and hence $h \in W_{l o c}^{1, x}$. Thus we can apply the argument above to show that $h_{x} \in B V_{\text {loc }}$.

Second Step. Shock admissibility.

By Vol'pert's chain rule (see [1]), the condition that the entropy dissipation must be non-positive translates into the following shock admissibility condition:

$$
-s\left[\eta\left(u^{+}\right)-\eta\left(u^{--}\right)\right]+q\left(u^{+}\right)-q\left(u^{-}\right) \leq 0
$$

for all convex entropy-cntropy flux pairs $(\eta, q)$ and for all points in the jump (shock) set $J_{u}$ of $u$. Here $u^{ \pm}$are the right (resp. left) trace of $u$ on $J_{u}$, and $s$ is the shock speed, i.e., the slope of the tangent to $J_{u}$ in $(t, x)$. We refer to Sec. 8.5 of [4]. Condition (2.8) follows from the fact that

$$
u^{+} \leq u^{-} \mathcal{H}^{1} \text {-a.e. on } J_{u} \text { (only decreasing jumps), }
$$

which itself is a consequence of $h$ being a viscosity solution. For simplicity, we check that (2.9) holds when $h$ is smooth outside a differentiable curve $\gamma:=\{(t, x(t)) \mid t \geq 0\}$. Indeed, by a standard blow-up argument, it is sufficient to prove (2.9) when $u$ is a piecewise constant function that jumps along a line.

Suppose that $h$ is given on the right- (resp. left-) hand side of $\gamma$ by smooth functions $h^{ \pm}$, satisfying $h_{t}^{ \pm}+f\left(h_{x}^{ \pm}\right)=0$. Assume $h$ is continuous along the curve, i.e., $h^{+}(t, x(t))=$ $h^{-}(t, x(t))$ for all $t \geq 0$. From $\frac{d}{d t} h^{+}(t, x(t))=\frac{d}{d t} h^{-}(t, x(t))$, after a straightforward calculation, we obtain

$$
-x^{\prime}(t)\left[h_{x}^{+}(t, x(t))-h_{x}^{-}(t, x(t))\right]+f\left(h_{x}^{+}(t, x(t))\right)-f\left(h_{x}^{-}(t, x(t))\right)=0
$$

for all $t \geq 0$ (Rankine-Hugoniot). Now fix some point $(T, X=x(T)) \in \gamma$. Then we claim

$$
h_{x}^{+}(T, X) \leq h_{x}^{-}(T, X)
$$

In fact, assume the opposite and fix some $c \in\left(h_{x}^{-}(T, X), h_{x}^{+}(T, X)\right)$. Consider the function

$$
\zeta(t, x)=h^{-}(t, x(t))+c(x-x(t)) .
$$

Then $h-\zeta$ has a local minimum at $(T, X)$. We compute

$$
\begin{aligned}
\zeta_{t}(T, X)+f\left(\zeta_{x}(T, X)\right) & =h_{t}^{-}(T, X)+x^{\prime}(T) h_{x}^{-}(T, X)-c x^{\prime}(T)+f(c) \\
& =-x^{\prime}(T)\left[c-h_{x}^{-}(T, X)\right]+f(c)-f\left(h_{x}^{-}(T, X)\right) .
\end{aligned}
$$

The r.h.s. is a strictly convex function in $c$ which vanishes in $h_{x}^{ \pm}(T, X)$ because of (2.10). Since $h_{x}^{-}(T, X)<c<h_{x}^{+}(T, X)$ by assumption, we have $\zeta_{t}(T, X)+f\left(\zeta_{x}(T, X)\right)<$ 0 . But that is a contradiction: $h$ is a viscosity solution. This gives the claim. 
3. Preliminary lemmas on averages of functions. To prove the theorems, we need a result on averages of functions.

Definition 3.1. Let $\mu$ be a probability measure on $\mathbf{R}$. For every vector-valued map $V \in L^{1}(\mathbf{R}, \mu)$. we set $\langle V\rangle:=\int_{\Omega} V(u) d \mu(u)$. Let $f . \eta \in W_{l o c}^{1 . x}(\mathbf{R})$ and $q(v):=$ $\int_{0}^{\prime \prime} f^{\prime}(\tau) \eta^{\prime}(\tau) d \tau$. If $\mu$ is compactly supported. then we define the bilinear form

$$
\begin{aligned}
B(f . \eta) & :=\left\langle\left(\begin{array}{l}
\eta \\
q
\end{array}\right) \cdot\left(\begin{array}{c}
-f \\
u
\end{array}\right)\right\rangle-\left\langle\left(\begin{array}{l}
\eta \\
q
\end{array}\right)\right\rangle \cdot\left\langle\left(\begin{array}{c}
-f \\
u
\end{array}\right)\right\rangle \\
& =\langle u q\rangle-\langle\eta f\rangle+\langle f\rangle\langle\eta\rangle-\langle u\rangle\langle q\rangle .
\end{aligned}
$$

When $\mu$ has noncompact support we define $B(f . \eta)$ whenever all the functions appearing in $(3.1)$ are $\mu$-simmmable.

In the definition above and in what follows, $u$ will always denote the independent real variable or the identity function on $\mathbf{R}$. For example. $\langle u\rangle$ and $\left\langle u^{2}\right\rangle$ denote $\int_{\mathbf{R}} u d \mu(u)$ and $\int_{\mathbf{R}} u^{2} d \mu(u)$.

Proposition 3.2. (a) If $u^{2}$ is $\mu$-simmanale, then $3 B\left(u^{2} \cdot u^{2}\right) \geq\left\langle(u-\langle u\rangle)^{4}\right\rangle$.

(b) If $\mu$ has compact support and $f$ and $\|$ are both convex, then $B(f . \eta) \geq 0$.

(c) If $\mu$ has compact support and $f^{\prime \prime} . \eta^{\prime \prime} \geq 2 c$. then $3 B(f . \eta) \geq c^{2}\left\langle(u-\langle u\rangle)^{4}\right\rangle$.

Proof. (a) Since $f(u)=\eta(u)=u^{2}$ and $q(u)=\frac{1}{3} u^{3}$. (3.1) implies

$$
\begin{aligned}
B\left(u^{2} \cdot u^{2}\right) & =\frac{4}{3}\left\langle u^{4}\right\rangle-\left\langle u^{4}\right\rangle+\left\langle u^{2}\right\rangle^{2}-\frac{4}{3}\langle u\rangle\left\langle u^{3}\right\rangle \\
& =\frac{1}{3}\left\langle u^{1}\right\rangle+\left\langle u^{2}\right\rangle^{2}-\frac{4}{3}\langle u\rangle\left\langle u^{3}\right\rangle .
\end{aligned}
$$

Recall that $\langle u\rangle^{2} \leq\left\langle u^{2}\right\rangle$ by .Jensen and thus

$$
0 \leq u^{2}-2 u\langle u\rangle+\langle u\rangle^{2} \leq u^{2}-2 u\langle u\rangle+\left\langle u^{2}\right\rangle \text {. }
$$

Hence we obtain

$$
\begin{aligned}
\left\langle(u-\langle u\rangle)^{4}\right\rangle & =\left\langle\left(u^{2}-2 u\langle u\rangle+\langle u\rangle^{2}\right)^{2}\right\rangle \leq\left\langle\left(u^{2}-2 u\langle u\rangle+\left\langle u^{2}\right\rangle\right)^{2}\right\rangle \\
& =\left\langle u^{4}\right\rangle-4\langle u\rangle\left\langle u^{3}\right\rangle+2\left\langle u^{2}\right\rangle^{2}+4\left\langle u^{2}\right\rangle\langle u\rangle^{2}-4\langle u\rangle^{2}\left\langle u^{2}\right\rangle+\left\langle u^{2}\right\rangle^{2} \\
& =\left\langle u^{4}\right\rangle-4\langle u\rangle\left\langle u^{3}\right\rangle+3\left\langle u^{2}\right\rangle^{2} \stackrel{(3.2)}{=} 3 B\left(u^{2} \cdot u^{2}\right) .
\end{aligned}
$$

(b) W.l.o.g. we may assume that $\|$ is smooth and $\eta^{\prime \prime}$ has compact support. Then we have

$$
\eta(u)=\int_{-\infty}^{+\infty} \frac{1}{2} \eta^{\prime \prime}(k)|u-k| d k+\text { const. }
$$

Hence we may assime that $\eta$ is of Kruzhkov's form

$$
\eta(u)=|u-k| \text { and thus } q(u)=\operatorname{sign}(u-k)(f(u)-f(k)) \text {. }
$$

We now have

$$
\begin{aligned}
B(f . \eta)= & \langle(u-k \cdot) q\rangle-\langle\eta(f(u)-f(k))\rangle+\langle f(u)-f(k)\rangle\langle\eta\rangle-\langle u-k \cdot\rangle\langle q\rangle \\
= & \langle f(u)-f(k)\rangle\langle\mid u-k \cdot\rangle-\langle u-k \cdot\rangle\langle\operatorname{sign}(u-k)(f(u)-f(k \cdot))\rangle \\
= & \left\langle f(u)-f(k)-f^{\prime}(k)(u-k)\right\rangle\langle|u-k \cdot|\rangle \\
& -\langle u-k \cdot\rangle\left\langle\operatorname{sign}(u-k)\left(f(u)-f(k)-f^{\prime}(k)(u-k \cdot)\right)\right\rangle .
\end{aligned}
$$


The latter expression is non-negative, since by convexity of $f$.

$$
f(u)-f(k)-f^{\prime}(k)(u-k) \geq 0 .
$$

(c) Consider the convex functions $f_{1}(v)=f(v)-c v^{2}$ and $\eta_{1}(v)=\eta(v)-c v^{2}$. Thanks to (b), $B\left(f_{1}, \eta_{1}\right), B\left(f_{1}, v^{2}\right)$, and $B\left(\imath^{2} \cdot \eta_{1}\right)$ are all non-negative. Hence (a) completes the proof.

4. Proof of Theorem 2.3. Note that $h$ is a Lipschitz function. The proof of the Theorem is split into two parts. In the first part, we prove that $h$ is a viscosity subsolution of $h_{t}+f\left(h_{x}\right)=0$. In the second part, we prove that $h$ is a viscosity supersolution.

4.1. Viscosity subsolution. We have by construction of $h$ :

$$
h_{t}=-f(u)=-f\left(h_{x}\right) \text { a.e. in } \Omega .
$$

Let $\xi \in C_{c}^{\infty}\left(\mathbf{R}^{2}\right)$ be non-negative with $\int_{\mathbf{R}^{2}} \xi d t d x=1$. Set $\xi_{\varepsilon}(t, x):=\frac{1}{\varepsilon^{2}} \xi\left(\frac{t}{\varepsilon} \cdot \frac{x}{\varepsilon}\right)$. By Jensen

$$
0=\left(h_{t}+f\left(h_{x}\right)\right) \star \xi_{\varepsilon} \geq h_{t} \star \xi_{\varepsilon}+f\left(h_{x} \star \xi_{\varepsilon}\right)=\left(h \star \xi_{\varepsilon}\right)_{t}+f\left(\left(h \star \xi_{\varepsilon}\right)_{x}\right) .
$$

Hence $h \star \xi_{\varepsilon}$ is a classical subsolution and thus also a viscosity subsolution (see Corollary I. 6 of [3]). Since $h$ is continuous, $h \star \xi_{\varepsilon}$ converges locally uniformly to $h$ as $\varepsilon \downarrow$ ). Thus also $h$ is a viscosity subsolution. by the stability result in Theorem I.1 of [3].

4.2. Viscosity supersolution. To prove that $h$ is a viscosity supersolution wo have to show the following fact: If $\zeta$ is a smooth function such that $h-\zeta$ has a minimum at some $(t . x) \in \Omega$, then $\left[\zeta_{t}+f\left(\zeta_{x}\right)\right](t, x) \geq 0$. For simplicity we assume that $(t . r)=(0.0)$ and $[h-\zeta](0,0)=0$. Moreover, we assume that the minimum is strict. Indeed, if we choose $\varepsilon>0$ and consider $\zeta^{\varepsilon}(t, x):=\zeta(t, x)+\varepsilon\left(t^{2}+x^{2}\right)$, then $\left[h-\zeta^{\varepsilon}\right]$ has a strict minimum at $(0,0)$ and $\left[\zeta_{t}^{\varepsilon}+f\left(\zeta_{x}^{\varepsilon}\right)\right](0,0)=\left[\zeta_{t}+f\left(\zeta_{x}\right)\right](0,0)$.

For any $\delta>0$ consider

$$
\Omega_{\delta}:=\text { connected component of }\{(t, x):[h-\zeta](t, x)<\delta\} \text { containing }(0.0) \text {. }
$$

Since $h$ is continuous and the origin is a strict minimum, $\Omega_{\delta}$ is an open set and $\operatorname{diam}\left(\Omega_{\delta}\right) \downarrow$ 0 as $\delta \downarrow 0$. We introduce the notation

$$
\langle g\rangle_{\delta}:=f_{\Omega_{\delta}} g(t, x) d t d x=\frac{1}{\left|\Omega_{\delta}\right|} \int_{\Omega_{\delta}} g(t, x) d t d x .
$$

By definition of $h$,

$$
\left\langle\left(\begin{array}{l}
\zeta_{t} \\
\zeta_{x}
\end{array}\right)\right\rangle_{\delta}=-\left\langle\left(\begin{array}{c}
(h-\zeta)_{t} \\
(h-\zeta)_{x}
\end{array}\right)\right\rangle_{\delta}+\left\langle\left(\begin{array}{c}
-f(u) \\
u
\end{array}\right)\right\rangle_{\delta}
$$

For $\delta$ sufficiently small, we have $\Omega_{\delta} \subset \subset B_{1}$. Thus

$$
\left\langle(h-\zeta)_{t}\right\rangle_{\delta}=f_{\Omega_{\delta}}(h-\zeta)_{t}=\frac{1}{\left|\Omega_{\delta}\right|} \int_{\Omega_{\delta}}(h-\zeta)_{t}=\frac{1}{\left|\Omega_{\delta}\right|} \int_{B_{1}}(\min \{h-\zeta-\delta .0\})_{t} .
$$

Since the function $\min \{h-\zeta-\delta, 0\}$ is continuous and identically zero on a neighborhood of $\partial B_{1}$, the right-hand side of (4.3) vanishes. The same argument applies to $\left\langle(h-\zeta)_{x}\right\rangle_{\delta}$. 
Hence, from (4.2) we get

$$
\left\langle\left(\begin{array}{l}
\zeta_{t} \\
\zeta_{x}
\end{array}\right)\right\rangle_{\delta}=\left\langle\left(\begin{array}{c}
-f(u) \\
u
\end{array}\right)\right\rangle_{\delta}
$$

On the other hand, we have

$$
\begin{aligned}
& \left\langle\left(\begin{array}{l}
\zeta_{t} \\
\zeta_{x}
\end{array}\right) \cdot\left(\begin{array}{c}
\eta(u) \\
q(u)
\end{array}\right)\right\rangle_{\delta} \\
& =\left\langle\left(\begin{array}{c}
-f(u) \\
u
\end{array}\right) \cdot\left(\begin{array}{l}
\eta(u) \\
q(u)
\end{array}\right)\right\rangle_{\delta}-\left\langle\left(\begin{array}{c}
(h-\zeta)_{t} \\
(h-\zeta)_{x}
\end{array}\right) \cdot\left(\begin{array}{l}
\eta(u) \\
q(u)
\end{array}\right)\right\rangle_{\delta}
\end{aligned}
$$

Note that

$$
\begin{aligned}
& -\left\langle\left(\begin{array}{c}
(h-\zeta)_{t} \\
(h-\zeta)_{x}
\end{array}\right) \cdot\left(\begin{array}{c}
\eta(u) \\
q(u)
\end{array}\right)\right\rangle_{\delta} \\
& =-\frac{1}{\left|\Omega_{\delta}\right|} \int_{B_{1}}\left(\begin{array}{c}
(\min \{h-\zeta-\delta, 0\})_{t} \\
(\min \{h-\zeta-\delta, 0\})_{x}
\end{array}\right) \cdot\left(\begin{array}{c}
\eta(u) \\
q(u)
\end{array}\right) \\
& =\frac{1}{\left|\Omega_{\delta}\right|} \int_{B_{1}} \min \{h-\zeta-\delta, 0\}\left([\eta(u)]_{t}+[q(u)]_{x}\right) \stackrel{(2.4)}{\geq} 0 .
\end{aligned}
$$

Thus we obtain from $(4.5)$

$$
\left\langle\left(\begin{array}{l}
\zeta_{t} \\
\zeta_{x}
\end{array}\right) \cdot\left(\begin{array}{l}
\eta(u) \\
q(u)
\end{array}\right)\right\rangle_{\delta} \geq\left\langle\left(\begin{array}{c}
-f(u) \\
u
\end{array}\right) \cdot\left(\begin{array}{c}
\eta(u) \\
q(u)
\end{array}\right)\right\rangle_{\delta}
$$

Therefore, with $C=C\left(\|u\|_{\infty}\right)$,

$$
\begin{aligned}
& \left\langle\left(\begin{array}{c}
-f(u) \\
u
\end{array}\right) \cdot\left(\begin{array}{c}
\eta(u) \\
q(u)
\end{array}\right)\right\rangle_{\delta}-\left\langle\left(\begin{array}{c}
-f(u) \\
u
\end{array}\right)\right\rangle_{\delta} \cdot\left\langle\left(\begin{array}{c}
\eta(u) \\
q(u)
\end{array}\right)\right\rangle_{\delta} \\
& \stackrel{(4.6)}{\leq}\left\langle\left(\begin{array}{c}
\zeta_{t} \\
\zeta_{x}
\end{array}\right) \cdot\left(\begin{array}{l}
\eta(u) \\
q(u)
\end{array}\right)\right\rangle_{\delta}-\left\langle\left(\begin{array}{c}
-f(u) \\
u
\end{array}\right)\right\rangle_{\delta} \cdot\left\langle\left(\begin{array}{l}
\eta(u) \\
q(u)
\end{array}\right)\right\rangle_{\delta} \\
& \stackrel{(4.4)}{=}\left\langle\left(\begin{array}{c}
\zeta_{t} \\
\zeta_{x}
\end{array}\right) \cdot\left(\begin{array}{c}
\eta(u) \\
q(u)
\end{array}\right)\right\rangle_{\delta}-\left\langle\left(\begin{array}{c}
\zeta_{t} \\
\zeta_{x}
\end{array}\right)\right\rangle_{\delta} \cdot\left\langle\left(\begin{array}{c}
\eta(u) \\
q(u)
\end{array}\right)\right\rangle_{\delta} \\
& \leq C \sup _{\Omega_{\delta}}\left|\left(\begin{array}{c}
\zeta_{t} \\
\zeta_{x}
\end{array}\right)-\left\langle\left(\begin{array}{c}
\zeta_{t} \\
\zeta_{x}
\end{array}\right)\right\rangle_{\delta}\right|
\end{aligned}
$$

We can now apply Proposition 3.2 and obtain

$$
\begin{aligned}
& \frac{c^{2}}{3}\left\langle\left(u-\langle u\rangle_{\delta}\right)^{4}\right\rangle_{\delta} \\
& \quad \leq\left\langle\left(\begin{array}{c}
-f(u) \\
u
\end{array}\right) \cdot\left(\begin{array}{l}
\eta(u) \\
q(u)
\end{array}\right)\right\rangle_{\delta}-\left\langle\left(\begin{array}{c}
-f(u) \\
u
\end{array}\right)\right\rangle_{\delta} \cdot\left\langle\left(\begin{array}{l}
\eta(u) \\
q(u)
\end{array}\right)\right\rangle_{\delta} \\
& \quad \leq C \sup _{\Omega_{\delta}}\left|\left(\begin{array}{c}
\zeta_{t} \\
\zeta_{x}
\end{array}\right)-\left\langle\left(\begin{array}{c}
\zeta_{t} \\
\zeta_{x}
\end{array}\right)\right\rangle_{\delta}\right| .
\end{aligned}
$$

Since $\zeta$ is smooth, we deduce

$$
\lim _{\delta \downarrow 0}\left\langle\left(u-\langle u\rangle_{\delta}\right)^{4}\right\rangle_{\delta}=0 .
$$


Since $f$ is Lipschitz on $\left[-\|u\|_{\infty},\|u\|_{\infty}\right]$, we have

$$
\left|\langle f(u)\rangle_{\delta}-f\left(\langle u\rangle_{\delta}\right)\right| \leq C\left\langle\left|u-\langle u\rangle_{\delta}\right|\right\rangle_{\delta} \leq C\left\langle\left(u-\langle u\rangle_{\delta}\right)^{4}\right\rangle_{\delta}^{1 / 4}
$$

so that (4.9) yields $\lim _{\delta \downarrow 0}\left|\langle f(u)\rangle_{\delta}-f\left(\langle u\rangle_{\delta}\right)\right|=0$.

In view of (4.4), this translates into

$$
\lim _{\delta \backslash 0}\left|-\left\langle\zeta_{t}\right\rangle_{\delta}-f\left(\left\langle\zeta_{x}\right\rangle_{\delta}\right)\right|=0
$$

Since $\zeta$ is smooth, this yields, as desired,

$$
-\zeta_{t}(0,0)-f\left(\zeta_{x}(0,0)\right)=0
$$

\section{Proof of Theorem 2.4.}

5.1. Continuity. Let $h$ be as in Theorem 2.4. Then $h$ is continuous.

REMARK 5.1. The following statement holds: If $h \in L_{l o c}^{1}\left(\mathbf{R}^{2}\right), h_{t} \in L_{l o c}^{2}\left(\mathbf{R}^{2}\right)$, and $h_{x} \in L_{l o c}^{4}\left(\mathbf{R}^{2}\right)$, then

$$
\sup _{\left(t_{0}, x_{0}\right),\left(t_{1}, x_{1}\right) \in K} \frac{\left|h\left(t_{1}, x_{1}\right)-h\left(t_{0}, x_{0}\right)\right|}{\left|t_{1}-t_{0}\right|^{1 / 5}+\left|x_{1}-x_{0}\right|^{1 / 3}}<\infty \quad \text { for every bounded } K \subset \subset \mathbf{R}^{2}
$$

However, in what follows, we give a simpler proof of the continuity of $h$, based on the additional information provided by (2.5) and (2.6).

Testing with a cut-off function $\eta$ in $x$, we obtain from (2.6) that $\int \frac{1}{2} \eta u^{2} d x$ is locally bounded in $t$, that is,

$$
u \in L_{l o c}^{\infty}\left(\mathbf{R}_{t}, L_{l o c}^{2}\left(\mathbf{R}_{x}\right)\right)
$$

On one hand, because of $h_{x}=u$, this yields Hölder continuity in $x$, uniformly in $t$, i.e.,

$$
h \in L_{l o c}^{\infty}\left(\mathbf{R}_{t}, C_{l o c}^{0, \frac{1}{2}}\left(\mathbf{R}_{x}\right)\right)
$$

On the other hand, because of $h_{t}=-\frac{1}{2} u^{2}$, this gives Lipschitz continuity in $t$ w.r.t. the $L^{1}$-norm in $x$ :

$$
h \in C_{l o c}^{0.1}\left(\mathbf{R}_{t}, L_{l o c}^{1}\left(\mathbf{R}_{x}\right)\right)
$$

We now argue "by interpolation" that (5.2) and (5.3) imply Hölder continuity in $t$, uniformly in $x$, i.e.,

$$
h \in L_{l o c}^{\infty}\left(\mathbf{R}_{x}, C_{l o c}^{0, \frac{1}{3}}\left(\mathbf{R}_{t}\right)\right)
$$


Indeed, let $\xi \in C_{c}^{x}\left(\mathbf{R}_{x}\right)$ be non-negative with supp $\xi \subset(-1,1)$ and $\int_{\mathbf{R}} \xi d x=1$. Set $\xi_{\varepsilon}(x):=\frac{1}{\varepsilon} \xi\left(\frac{x}{\varepsilon}\right)$ and let $\star$ denote the convolution in the r-variable. We have

$$
\begin{aligned}
& \left|h\left(t_{1}, x\right)-h\left(t_{2}, x\right)\right| \\
& \leq\left|h\left(t_{1} . . x\right)-\left(h \star \xi_{\xi}\right)\left(t_{1}, x\right)\right|+\left|\left(h \star \xi_{\varepsilon}\right)\left(t_{1} . x\right)-\left(h \star \xi_{\varepsilon}\right)\left(t_{2}, x\right)\right| \\
& +\left|\left(h \star \xi_{z}\right)\left(t_{2} . x\right)-h\left(t_{2} . x\right)\right| \\
& \leq \quad \varepsilon^{1 / 2} \operatorname{siu}_{y \cdot|x-y| \leq \varepsilon} \frac{\left|h\left(t_{1} . x\right)-h\left(t_{1} \cdot y\right)\right|}{|r-y|^{1 / 2}} \\
& +\operatorname{sip}_{\mathbf{R}}|\xi| \cdot \frac{1}{\varepsilon} \int_{x-\varepsilon}^{x+\varepsilon}\left|h\left(t_{1} \cdot y\right)-h\left(t_{2} \cdot y\right)\right| d y \\
& +\varepsilon^{1 / 2} \sup _{y,|x-y| \leq \varepsilon} \frac{\left|h\left(t_{2}, x\right)-h\left(t_{2}, y\right)\right|}{|x-y|^{1 / 2}} \\
& \stackrel{(5.2) \cdot(5.3)}{\leq} C\left(\varepsilon^{1 / 2}+\frac{\left|t_{1}-t_{2}\right|}{\varepsilon}\right)
\end{aligned}
$$

Choosing $\Xi=\left|t_{1}-t_{2}\right|^{2 / 3}$ richds (5.4).

5.2. Viscosity subsolution. Since $h$ is continuous. the fact that $h$ is a viscosity subsolution follows from the argument given in Subsection 4.1 with $f(u)=u^{2} / 2$.

5.3. Viscosity supersolution. The goal is proving the following

Proposition 5.2. Let $\zeta$ be a smooth function with $\zeta(0,0)=0$ and such that $h-\zeta$ has a minimum in $(0.0)$. Then $\left[\zeta_{t}(0.0)+\frac{1}{2} \zeta_{. r}^{2}(0.0)\right]=0$.

To simplify the notation. we will write $g(\varepsilon . \delta) \lesssim h(\varepsilon . \delta)$ whenever there exist constants $C_{1}, C_{2}>0$ such that $g(\varepsilon . \delta) \leq C_{1} h(\varepsilon . \delta)$ for $|\delta| .|\varepsilon| \leq C_{2}$.

Proof. Without loss of generality we may assume $h(0.0)=\zeta(() .0)=0$. For $0<\Xi \leq 1$. we set $\zeta_{\varepsilon}:=\zeta-\xi|(t . r)|$. Then $h-\zeta_{\varepsilon}$ has a strict minimm at 0 . Given $\delta>0$. we introduce the notation

$$
\Omega_{\varepsilon . \delta}:=\left\{(t . . r):\left[h-\zeta_{\varepsilon}\right](t . . r)<\delta\right\} .
$$

Since $h-\zeta_{\varepsilon} \geq[h-\zeta](t, x)+\varepsilon|(t, x)| \geq[h-\zeta](0.0)+\varepsilon|(t, x)|=\varepsilon|(t, x)|$, we have

$$
\Omega_{\varepsilon . \delta} \subset\{(t . x): \varepsilon|(t . x)|<\delta\}=B_{\delta / \varepsilon}((0.0)) .
$$

We introduce the notation

$$
\langle u\rangle_{\varepsilon . \delta}:=\frac{1}{\left|\Omega_{\varepsilon . \delta}\right|} \int_{\Omega_{\varepsilon, \delta}} u(t . . x) d t d . x .
$$

First Step. We start by obscrving:

$$
\left\langle\left(\begin{array}{c}
\zeta_{\varepsilon t} \\
\zeta_{\varepsilon, r}
\end{array}\right)\right\rangle_{\varepsilon . \delta}=\left\langle\left(\begin{array}{c}
-\frac{1}{2} u^{2} \\
u
\end{array}\right)\right\rangle_{\varepsilon . \delta} .
$$

Indeed

$$
\left\langle\left(\begin{array}{c}
-\frac{1}{2} u^{2} \\
u
\end{array}\right)\right\rangle_{\varepsilon, \delta}-\left\langle\left(\begin{array}{c}
\zeta_{\varepsilon t} \\
\zeta_{\varepsilon, x}
\end{array}\right)\right\rangle_{\varepsilon, \delta}=\left\langle\left(\begin{array}{c}
\left(h-\zeta_{\varepsilon}\right)_{t} \\
\left(h-\zeta_{\varepsilon}\right)_{x}
\end{array}\right)\right\rangle_{\varepsilon, \delta} .
$$


Note that

$$
\left\langle h-\zeta_{\varepsilon t}\right\rangle_{\varepsilon . \delta}=\frac{1}{\left|\Omega_{\varepsilon, \delta}\right|} \int_{\mathbf{R}^{2}}\left(\min \left\{h-\zeta_{\varepsilon}-\delta, 0\right\}\right)_{t}=0 .
$$

and an analogous computation gives $\left\langle\left(h-\zeta_{\varepsilon}\right)_{x}\right\rangle_{\varepsilon . \delta}=0$. This establishes (5.6). An easy consequence of $(5.6)$ is

$$
\left\langle u^{2}\right\rangle_{\varepsilon . \delta}=\left\langle\zeta_{\varepsilon t}\right\rangle_{\varepsilon . \delta} \lesssim 1
$$

Jensen inequality gives $\langle|u|\rangle_{\varepsilon, \delta} \lesssim 1$. Thus we get

$$
\left\langle\left|\left(\begin{array}{c}
h_{t} \\
h_{x}
\end{array}\right)\right|\right\rangle_{\varepsilon, \delta} \lesssim 1
$$

This translates into

$$
\left\langle\left|\left(\begin{array}{c}
\left(h-\zeta_{\varepsilon}\right)_{t} \\
\left(h-\zeta_{\varepsilon}\right)_{x}
\end{array}\right)\right|\right\rangle_{\varepsilon . \delta} \leq\left\langle\left|\left(\begin{array}{c}
h_{t} \\
h_{x}
\end{array}\right)\right|\right\rangle_{\varepsilon . \delta}+\left\langle\left|\left(\begin{array}{c}
\zeta_{\varepsilon t} \\
\zeta_{\varepsilon x}
\end{array}\right)\right|\right\rangle_{\varepsilon . \delta} \lesssim 1
$$

Second Step. We now prove that

$$
\delta^{2} \lesssim\left|\Omega_{\varepsilon, \delta}\right|
$$

Indeed, (5.8) can be reformulated as

$$
\int_{\mathbf{R}^{2}}\left|\left(\begin{array}{c}
\left(\min \left\{h-\zeta_{\varepsilon}-\delta, 0\right\}\right)_{t} \\
\left(\min \left\{h-\zeta_{\varepsilon}-\delta, 0\right\}\right)_{x}
\end{array}\right)\right|=\int_{\Omega_{\varepsilon, \delta}}\left|\left(\begin{array}{c}
\left(h-\zeta_{\varepsilon}\right)_{t} \\
\left(h-\zeta_{\varepsilon}\right)_{x}
\end{array}\right)\right| \lesssim\left|\Omega_{\varepsilon, \delta}\right| .
$$

By (5.10) and Sobolev inequality,

$$
\left[\int_{\mathbf{R}^{2}}\left(\min \left\{h-\zeta_{\varepsilon}-\delta, 0\right\}\right)^{2}\right]^{1 / 2} \lesssim\left|\Omega_{\varepsilon . \delta}\right|
$$

Hölder inequality yiclds

$$
-\int_{\mathbf{R}^{2}} \min \left\{h-\zeta_{\varepsilon}-\delta, 0\right\} \lesssim\left|\Omega_{\varepsilon, \delta}\right|^{1 / 2}\left[\int_{\mathbf{R}^{2}}\left(\min \left\{h-\zeta_{\varepsilon}-\delta, 0\right\}\right)^{2}\right]^{1 / 2} \lesssim\left|\Omega_{\varepsilon, \delta}\right|^{3 / 2} .
$$

Note that

$$
I(\delta):=-\int_{\mathbf{R}^{2}} \min \left\{h-\zeta_{\varepsilon}-\delta, 0\right\}=\int_{0}^{\delta}\left|\Omega_{\varepsilon, s}\right| d s .
$$

Thus we obtain the differential inequality

$$
I(\delta) \stackrel{(5.12)}{\lesssim}\left|\Omega_{\varepsilon, \delta}\right|^{3 / 2}=\left[\frac{d}{d \delta} I(\delta)\right]^{3 / 2} .
$$

Since $I(\delta)>0$ for $\delta>0$, we get $1 \lesssim \frac{d}{d \delta}\left[(I(\delta))^{1 / 3}\right]$, which yields $\delta^{3} \lesssim I(\delta)$. Note that $\left|\Omega_{\varepsilon, \delta}\right|$ is a non-decreasing function of $\delta$. Hence,

$$
\delta\left|\Omega_{\varepsilon, \delta}\right| \geq \int_{0}^{\delta}\left|\Omega_{\varepsilon, s}\right| d s \gtrsim \delta^{3}
$$

which establishes (5.9).

Third Step. We now argue that

$$
\left\langle\left(\begin{array}{c}
-u^{2} / 2 \\
u
\end{array}\right) \cdot\left(\begin{array}{c}
u^{2} / 2 \\
u^{3} / 3
\end{array}\right)\right\rangle_{\varepsilon, \delta}-\left\langle\left(\begin{array}{c}
\zeta_{\varepsilon t} \\
\zeta_{\varepsilon x}
\end{array}\right) \cdot\left(\begin{array}{c}
u^{2} / 2 \\
u^{3} / 3
\end{array}\right)\right\rangle_{\varepsilon . \delta} \lesssim \frac{\mu\left(B_{\delta / \varepsilon}(0,0)\right)}{\delta} .
$$


Indeed, we have

$$
\begin{aligned}
& \left\langle\left(\begin{array}{c}
-u^{2} / 2 \\
u
\end{array}\right) \cdot\left(\begin{array}{c}
u^{2} / 2 \\
u^{3} / 3
\end{array}\right)\right\rangle_{\varepsilon . \delta}-\left\langle\left(\begin{array}{c}
\zeta_{\varepsilon t} \\
\zeta_{\varepsilon . x}
\end{array}\right) \cdot\left(\begin{array}{c}
u^{2} / 2 \\
u^{3} / 3
\end{array}\right)\right\rangle_{\varepsilon, \delta} \\
& =\left\langle\left(\begin{array}{c}
\left(h-\zeta_{\varepsilon}\right)_{t} \\
\left(h-\zeta_{\varepsilon}\right)_{x}
\end{array}\right) \cdot\left(\begin{array}{c}
u^{2} / 2 \\
u^{3} / 3
\end{array}\right)\right\rangle_{\varepsilon, \delta} \\
& =\frac{1}{\left|\Omega_{\varepsilon, \delta}\right|} \int_{\Omega_{\varepsilon, \delta}}\left(\begin{array}{c}
\left(h-\zeta_{\varepsilon}\right)_{t} \\
\left(h-\zeta_{\varepsilon}\right)_{x}
\end{array}\right) \cdot\left(\begin{array}{c}
u^{2} / 2 \\
u^{3} / 3
\end{array}\right) \\
& =\frac{1}{\left|\Omega_{\varepsilon, \delta}\right|} \int_{\mathbf{R}^{2}}\left(\begin{array}{c}
\left(\min \left\{h-\zeta_{\varepsilon}-\delta .0\right\}\right)_{t} \\
\left(\min \left\{h-\zeta_{\varepsilon}-\delta .0\right\}\right)_{x}
\end{array}\right) \cdot\left(\begin{array}{c}
u^{2} / 2 \\
u^{3} / 3
\end{array}\right) \\
& =\frac{1}{\left|\Omega_{\varepsilon, \delta}\right|} \int_{\mathbf{R}^{2}}\left(-\min \left\{h-\zeta_{\varepsilon}-\delta, 0\right\}\right)\left(\left(\frac{u^{2}}{2}\right)_{t}+\left(\frac{u^{3}}{3}\right)_{x}\right) \\
& \leq \frac{1}{\left|\Omega_{\varepsilon . \delta}\right|} \int\left(-\min \left\{h-\zeta_{\varepsilon}-\delta, 0\right\}\right) d \mu \leq \frac{\delta}{\left|\Omega_{\varepsilon . \delta}\right|} \mu\left(\Omega_{\varepsilon, \delta}\right) \\
& \leq \frac{\delta}{\left|\Omega_{\varepsilon, \delta}\right|} \mu\left(B_{\delta / \varepsilon}((0,0))\right) \stackrel{(5.9)}{\lesssim} \frac{\mu\left(B_{\delta / \varepsilon}((0,0))\right)}{\delta} .
\end{aligned}
$$

This establishes (5.13).

Fourth Step. We now prove

$$
\left\langle u^{4}\right\rangle_{\varepsilon, \delta} \lesssim \varepsilon^{-1} \text { and }\left\langle|u|^{3}\right\rangle_{\varepsilon . \delta} \lesssim \varepsilon^{-1 / 2}
$$

Indeed

$$
\begin{aligned}
\left\langle u^{4}\right\rangle_{\varepsilon, \delta} & \lesssim\left\langle\left(\begin{array}{c}
-u^{2} / 2 \\
u
\end{array}\right) \cdot\left(\begin{array}{c}
u^{2} / 2 \\
u^{3} / 3
\end{array}\right)\right\rangle_{\varepsilon . \delta} \\
& \lesssim\left\langle\left(\begin{array}{c}
\zeta_{\varepsilon t} \\
\zeta_{\varepsilon x}
\end{array}\right) \cdot\left(\begin{array}{c}
u^{2} / 2 \\
u^{3} / 3
\end{array}\right)\right\rangle_{\varepsilon . \delta}+\frac{\mu\left(B_{\delta / \varepsilon}((0,0))\right)}{\delta} \\
& \lesssim \sup _{\Omega_{\varepsilon . \delta}}\left|\left(\begin{array}{c}
\zeta_{\varepsilon t} \\
\zeta_{\varepsilon x}
\end{array}\right)\right|\left\langle\left|\left(\begin{array}{c}
u^{2} / 2 \\
u^{3} / 3
\end{array}\right)\right|\right\rangle_{\varepsilon . \delta}+\frac{1}{\varepsilon}\left[\frac{\varepsilon}{\delta} \mu\left(B_{\delta / \varepsilon}((0,0))\right)\right] \\
& \lesssim\left\langle u^{2}\right\rangle_{\varepsilon . \delta}+\left\langle|u|^{3}\right\rangle_{\varepsilon, \delta}+\varepsilon^{-1} \stackrel{(5.7)}{\lesssim} 1+\left[\left\langle u^{2}\right\rangle_{\varepsilon, \delta}\right]^{1 / 2}\left[\left\langle u^{4}\right\rangle_{\varepsilon . \delta}\right]^{1 / 2}+\varepsilon^{-1} .
\end{aligned}
$$

By Young's inequality, this yields $\left\langle u^{4}\right\rangle_{\varepsilon . \delta} \lesssim \varepsilon^{-1}$. Hölder inequality gives

$$
\left\langle|u|^{3}\right\rangle_{\varepsilon, \delta} \leq\left[\left\langle u^{4}\right\rangle_{\varepsilon, \delta}\right]^{1 / 2}\left[\left\langle u^{2}\right\rangle_{\varepsilon, \delta}\right]^{1 / 2} \lesssim \varepsilon^{-1 / 2}
$$

Fifth Step. We now argue that

$$
\left|\left\langle\left(\begin{array}{c}
\zeta_{\varepsilon t} \\
\zeta_{\varepsilon x}
\end{array}\right) \cdot\left(\begin{array}{c}
u^{2} / 2 \\
u^{3} / 3
\end{array}\right)\right\rangle_{\varepsilon, \delta}-\left\langle\left(\begin{array}{c}
\zeta_{\varepsilon t} \\
\zeta_{\varepsilon x}
\end{array}\right)\right\rangle_{\varepsilon, \delta} \cdot\left\langle\left(\begin{array}{c}
u^{2} / 2 \\
u^{3} / 3
\end{array}\right)\right\rangle_{\varepsilon, \delta}\right| \lesssim \varepsilon^{1 / 2}+\frac{\delta}{\varepsilon^{3 / 2}} .
$$


Indeed

$$
\begin{aligned}
& \left|\left\langle\left(\begin{array}{c}
\zeta_{\varepsilon t} \\
\zeta_{\varepsilon x}
\end{array}\right) \cdot\left(\begin{array}{c}
u^{2} / 2 \\
u^{3} / 3
\end{array}\right)\right\rangle_{\varepsilon, \delta}-\left\langle\left(\begin{array}{c}
\zeta_{\varepsilon t} \\
\zeta_{\varepsilon x}
\end{array}\right)\right\rangle_{\varepsilon, \delta} \cdot\left\langle\left(\begin{array}{c}
u^{2} / 2 \\
u^{3} / 3
\end{array}\right)\right\rangle_{\varepsilon, \delta}\right| \\
& \leq \quad \sup _{\Omega_{\varepsilon, \delta}}\left|\left(\begin{array}{c}
\zeta_{\varepsilon t} \\
\zeta_{\varepsilon x}
\end{array}\right)-\left\langle\left(\begin{array}{c}
\zeta_{\varepsilon t} \\
\zeta_{\varepsilon x}
\end{array}\right)\right\rangle_{\varepsilon, \delta}\right|\left\langle\left|\left(\begin{array}{c}
u^{2} / 2 \\
u^{3} / 3
\end{array}\right)\right|\right\rangle_{\varepsilon, \delta} \\
& \leq \quad\left[\begin{array}{c}
\operatorname{Osc} \\
\Omega_{\varepsilon, \delta}
\end{array}\left(\begin{array}{c}
\zeta_{\varepsilon t} \\
\zeta_{\varepsilon x}
\end{array}\right)\right]\left(\left\langle u^{2}\right\rangle_{\varepsilon, \delta}+\left\langle|u|^{3}\right\rangle_{\varepsilon, \delta}\right) \\
& \leq \quad\left[\underset{\Omega_{\varepsilon, \delta}}{\operatorname{osc}}\left(\begin{array}{c}
\zeta_{t} \\
\zeta_{x}
\end{array}\right)+2 \varepsilon\right]\left(\left\langle u^{2}\right\rangle_{\varepsilon, \delta}+\left\langle|u|^{3}\right\rangle_{\varepsilon, \delta}\right) \\
& \stackrel{(5.5) .(5.7),(5.14)}{\lesssim}\left(\frac{\delta}{\varepsilon}+\varepsilon\right)\left(1+\varepsilon^{-1 / 2}\right) \lesssim \frac{\delta}{\varepsilon^{3 / 2}}+\varepsilon^{1 / 2} .
\end{aligned}
$$

Sixth Step. Combining (5.6), (5.13), and (5.15), we get

$$
\begin{gathered}
\left\langle\left(\begin{array}{c}
-u^{2} / 2 \\
u
\end{array}\right) \cdot\left(\begin{array}{c}
u^{2} / 2 \\
u^{3} / 3
\end{array}\right)\right\rangle_{\varepsilon, \delta}-\left\langle\left(\begin{array}{c}
-u^{2} / 2 \\
u
\end{array}\right)\right\rangle_{\varepsilon, \delta} \cdot\left\langle\left(\begin{array}{c}
u^{2} / 2 \\
u^{3} / 3
\end{array}\right)\right\rangle_{\varepsilon, \delta} \\
\lesssim \frac{\delta}{\varepsilon^{3 / 2}}+\varepsilon^{1 / 2}+\frac{\mu\left(B_{\delta / \varepsilon}((0,0))\right)}{\delta}
\end{gathered}
$$

According to Proposition 3.2 (b), we obtain

$$
\left\langle\left(u-\langle u\rangle_{\varepsilon, \delta}\right)^{4}\right\rangle_{\varepsilon, \delta} \lesssim \frac{\delta}{\varepsilon^{3 / 2}}+\varepsilon^{1 / 2}+\frac{\mu\left(B_{\delta / \varepsilon}((0,0))\right)}{\delta} .
$$

On the other hand, we have

$$
\begin{aligned}
\left|\zeta_{t}(0,0)+\frac{1}{2} \zeta_{x}^{2}(0,0)\right| & \stackrel{(5.5)}{\lesssim}\left|\left\langle\zeta_{t}\right\rangle_{\varepsilon, \delta}+\frac{1}{2}\left[\left\langle\zeta_{x}\right\rangle_{\varepsilon, \delta}\right]^{2}\right|+\frac{\delta}{\varepsilon} \\
& \lesssim\left|\left\langle\zeta_{\varepsilon t}\right\rangle_{\varepsilon, \delta}+\frac{1}{2}\left[\left\langle\zeta_{\varepsilon x}\right\rangle_{\varepsilon, \delta}\right]^{2}\right|+\varepsilon+\frac{\delta}{\varepsilon} \\
& \stackrel{(5.6)}{=}\left|-\left\langle\frac{u^{2}}{2}\right\rangle_{\varepsilon, \delta}+\frac{1}{2}\left[\langle u\rangle_{\varepsilon, \delta}\right]^{2}\right|+\varepsilon+\frac{\delta}{\varepsilon} \\
& =\frac{1}{2}\left\langle\left(u-\langle u\rangle_{\varepsilon, \delta}\right)^{2}\right\rangle_{\varepsilon, \delta}+\varepsilon+\frac{\delta}{\varepsilon} \\
& \lesssim\left[\left\langle\left(u-\langle u\rangle_{\varepsilon, \delta}\right)^{4}\right\rangle_{\varepsilon, \delta}\right]^{1 / 2}+\varepsilon+\frac{\delta}{\varepsilon} \\
& \lesssim\left(\frac{\delta .17)}{\varepsilon^{3 / 2}}+\varepsilon^{1 / 2}+\frac{\mu\left(B_{\delta / \varepsilon}((0,0))\right)}{\delta}\right)^{1 / 2}+\varepsilon+\frac{\delta}{\varepsilon}
\end{aligned}
$$

Letting first $\delta$ and then $\varepsilon$ go to 0 , we get $\zeta_{t}(0,0)+\frac{1}{2} \zeta_{x}^{2}(0,0)=0$. 


\section{REFERENCES}

[1] L. Ambrosio, N. Fusco, D. Pallara, Functions of bounded variation and free discontinuity problems. Oxford Mathematical Monographs. Clarendon Press. Oxford. 2000.

[2] L. Ambrosio, M. Lecumberry. T. Rivière, A viscosity property of minimizing micromagnetic configurations. Comm. Pure Appl. Nath. 56 (2003). pp. 681-688.

[3] M. G. Crandall, P. L. Lions. Viscosity solutions of Hamilton-Jacobi equations, Trans. Amer. Math. Soc. 277 (1983), pp. 1-42.

[4] C. Dafermos, Hyperbolic conservation laws in continuum physics. volume 325 of Grundlehren der Mathematischen Wissenschaften, Springer-Verlag, Berlin, 2000.

[5] L. Giacomelli, F. Otto, New bounds for the Kuramoto-Shivashinsky equation, to appear in Comm. Pure Appl. Math.

[6] S. N. Kruzhkov, First order quasilinear equations in several independent variables, Math. Sb. 123 (1970), pp. 228-255; English transl. in Math. USSR Sbornik 10 (1970). pp. 217-243.

[7] P. L. Lions, Generalized Solutions of Hamilton-Jacobi Equations, volume 69 of Research Notes in Mathematics. Pitman Advanced Publishing Program, London. 1982.

[8] O. A. Oleinik, Discontinuous solutions of nonlinear differential equations, Usp. Mat. Nauk. 12 (1957), pp. 3-73; English transl. in AMS Transl. 26 (1963). pp. 1155-1163.

[9] E. Y. Panov, Uniqueness of the Cauchy problem for a first order quasilinear equation with one admissible strictly convex entropy, Mat. Zametki 55 (1994). pp. 116-129, 159; English transl. in Math. Notes 55 (1994), pp. 517-525.

[10] B. Riemann, Über die Fortpflanzung ebener Luftwellen von endlicher Schwingungsweite. Abh. d. Königl. Ges. d. Wiss. zu Göttingen, Bd. 8 (1858/59) (Math. Cl.). pp. 43-65. 